La práctica del alli kawsay desde la resistencia. Caso de Zhiña, Ecuador

Ana María A. Ferrer, José Efraín Astudillo Banegas y Juan Sebastián Martines Tola

Pp. $81-104$

\title{
LA PRÁCTICA DEL ALLI KAWSAY DESDE LA RESISTENCIA. CASO DE ZHIÑA, ECUADOR
}

\author{
Resistance: the practice of alli kawsay. The case of Zhiña, Ecuador
}

\section{RESUMEN}

El presente artículo muestra los resultados de una investigación en la comuna indígena de Zhiña en el cantón de Nabón, Ecuador a partir del estudio de los discursos y prácticas alternativas al modelo de desarrollo clásico. El sumak kawsay entendido como vida hermosa, plena y digna, así como el alli kawsay, entendido como vida buena, son dos conceptos que representan la confrontación de los pueblos indígenas al modelo de desarrollo contemporáneo. En la historia de la comuna de Zhiña desde la compra de la tierra, la organización y negociación política ha sido el hilo conductor de la historia de la comuna. La confrontación se dio incluso al interior de la comuna a nivel identitario con los colonos, personas de la comuna que luego de migrar del lugar regresan a ella con visiones que entran en conflicto con las que históricamente identifican a Zhiña. La metodología empleada en la investigación fue de tipo cualitativa y participativa a partir de entrevistas en profundidad, observación y talleres.

Palabras claves: alli kawsay, sumak kawsay, resistencia indígena, lucha política, Zhiña.

\section{UNIVERSUM}

Revista de Humanidades y Ciencias Sociales

ANA MARÍA A. FERRER

Estudiante del Programa de Doctorado en Estudios Americanos de la Universidad de Santiago de Chile. Correo electrónico: ana. alonso@usach.cl

\section{JOSÉ EFRAÍN ASTUDILLO BANEGAS}

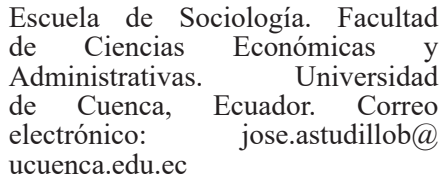

\section{JUAN SEBASTIÁN MARTINES TOLA}

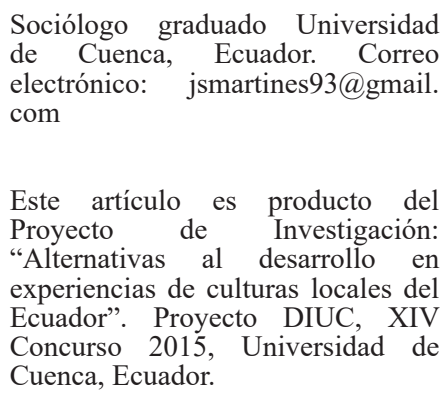
experiencias de culturas locales del Ecuador". Proyecto DIUC, XIV Concurso 2015, Universidad de Cuenca, Ecuador. 


\begin{abstract}
This article shows the results of an investigation in the territory of indigenous peoples from Zhiña, in the canton of Nabón, Ecuador, based on the study of the discourses and alternative practices of the orthodox development model. The sumak kawsay understood as a beautiful life and good living, as well as the alli kawsay, understood as a good life, are two concepts that represent the confrontation of indigenous peoples to the orthodox development model. In the history of the indigenous peoples from Zhiña since the purchase of land, the organization and political negotiation has been the guiding thread of the history of the territory. The confrontation occurred even within the indigenous people's territory at an identity level with the settlers, indigenous peoples who, after migrating from the indigenous territory, returned to it with visions that conflict with those that historically identify Zhiña. The methodology used in the research was qualitative and participatory, based on in-depth interviews, observation and workshops.
\end{abstract}

Keywords: alli kawsay, sumak kawsay, indigenous resistance, political struggle, Zhiña.

\title{
INTRODUCCIÓN
}

El propósito principal de este trabajo es presentar cómo la población de la comuna de Zhiña, en el cantón Nabón, Ecuador, expresa y construye su cotidianidad en el sumak kawsay, a partir de la confrontación al modelo clásico de contemporáneo. ${ }^{1}$ Es desde la articulación política que se encuentra una resistencia con otros actores que van desde la propia figura del Estado hasta habitantes de la comuna misma. Los resultados obtenidos fueron a partir de una metodología cualitativa y participativa realizada con entrevistas a profundidad, observación participante y talleres.

El punto de partida en el análisis de los datos es que existen muchas

1 Ver (Gudynas, 2011) para un desarrollo más amplio de este concepto. 
concepciones acerca de lo que es el sumak kawsay, así como de la existencia de consecuencias negativas derivadas del modelo de desarrollo clásico.

El modelo de desarrollo existe en el modus operandi de la sociedad ecuatoriana en espacios rurales y urbanos pese a las diferencias entre ambos espacios y una convergencia con elementos característicos del modelo desarrollista pese al cuestionamiento del mismo. En el caso de la comuna de Ecuador esta práctica alternativa viene construida a partir de la lucha política con el fin de mantener su identidad y formas de vida. Por tanto, hay una disputa entre formas de vida locales y formas de vida más características del desarrollo clásico en una misma escala local. Esta disputa se resuelve principalmente a través de propuestas políticas locales articuladas en distintos niveles territoriales.

\section{SOBRE EL CONCEPTO DE SUMAK KAWSAY - BUEN VIVIR}

El concepto sumak kawsay traducido al español como buen vivir, surge como propuesta frente al modelo de desarrollo convencional desde los pueblos andinos y de la Amazonía ecuatoriana. En el caso ecuatoriano, es plasmado en la Constitución de 2008, la cual en los artículos 71-74 recoge los derechos propios, así como de restauración, precaución, restricción, beneficio y apropiación de la naturaleza. (Constitución Ecuador, 2008). Este marco legal responde, en parte, a una lucha de los pueblos indígenas iniciada en la década de los 90 cuando, con aspiraciones y deseos de construir una sociedad diferente, se institucionaliza otra propuesta de modelo de desarrollo en la que son contempladas las vivencias de las diferentes culturas ancestrales. La creación de la CONAIE (Confederación de Nacionales Indígenas del Ecuador) y de su brazo político Pachakutik-Nuevo País, fueron indispensables en la creación del pensamiento detrás del buen vivir y de su viabilidad política (Vega, 2014).

El sumak kawsay hace referencia a una forma de vida y convivencia en armonía con la naturaleza y con otros seres humanos y se apoya en los principios de equidad social y sustentabilidad ambiental (Cubillo-Guevara \& 
Hidalgo-Capitán, 2015) Es por ello que la propuesta del buen vivir se levanta desde la crítica a la modernidad y al sistema capitalista que promueve el progreso como un proceso de acumulación económica independientemente de las consecuencias que este tiene a nivel social, cultural y ambiental.

El buen vivir plantea la revalorización de las culturas ancestrales, y sus buenas prácticas de vida armónica con la naturaleza. Esta concepción plantea una ruptura con la idea de desarrollo convencional en tanto que el desarrollo convencional aplica una fórmula universal para que un país o comunidad alcance la categoría de desarrollo. Por otro lado, el buen vivir al tener en cuenta las condiciones del territorio para cubrir las necesidades locales y colectivas y no el aprovechamiento máximo del mismo, rompe con la concepción dicotómica de desarrollado-subdesarrollado, ya que el desarrollo convencional deja de ser tomado en cuenta como un fin universal y, por ende, no se concibe la existencia de un único proceso por el que todos los países no desarrollados deban pasar. Esta visión dicotómica de las sociedades, conocida como la teoría de la modernización, es una concepción teórica que fue cuestionada desde Europa y América Latina en los años 70 (Joas \& Knöbl, 2016).

Es desde la cosmovisión de los pueblos indígenas que se presenta esta ruptura con la propuesta de desarrollo. Otra de las ideas que este modelo plantea es la relación antropocéntrica con la naturaleza, lo cual conlleva a una instrumentalización orientada a sostener un patrón de consumo que no considera los ciclos vitales de los seres vivos no humanos. El estilo de vida antropocéntrico supone cubrir necesidades que no necesariamente son tales, es decir, si tenemos en cuenta que las necesidades son pocas, finitas y clasificables en cualquier tiempo y espacio (Max-Neef M., 1993) las prácticas de consumo no estarían sustentadas en necesidades reales. Otro estilo de vida que normalmente es característico de comunidades que entran en disputa con el modelo de desarrollo es el biocentrismo (Gudynas, 2010) el que supone un choque de imaginarios sociales al considerar a la naturaleza como sujeto de derechos.

Si bien el sumak kawsay no se puede tomar como algo universal, sí se 
encuentran elementos que resultan básicos para entender a qué otra práctica de vida se está tratando de acercar, entre ellos: armonía con la naturaleza relacionada con el biocentrismo y la idea de autosuficiencia que reconoce solo algunas de las necesidades humanas (Acosta, 2010).

Las sociedades están por su dinamismo propio, en constaste cambio, sin embargo, los cambios sociales producidos en sociedades como la ecuatoriana se relacionan con la llegada de procesos económicos y sociales externos. Desde la teoría de la colonialidad se hace referencia a este tipo de sociedades latinoamericanas que son fruto de procesos de colonialidad objetiva y subjetiva, tales como la colonialidad del poder, la del saber y el ser (Restrepo \& Rojas, 2010).

Yo dudo que existan muchas comunidades donde uno pueda decir: ese es el buen vivir prístino, intocado. Porque la influencia colonial, 500 años de conquista, no termina cuando se van los españoles, continúa hasta ahora, no pasan y por eso es importante aproximarse teniendo conciencia de esa realidad y no caer nunca en la trampa de idealizar lo que propone el Buen Vivir o la vida del Buen Vivir (Acosta, 2016).

Si bien la teoría de la colonialidad plantea que los cambios sociales que se siguen sucediendo en estos territorios, están caracterizados en la aceptación de dimensiones relacionadas con la modernidad, se reconoce también la existencia de otros elementos que son propios de los sujetos que han sido históricamente encubiertos por la misma (Dussel, 2008). Esta disputa por el reconocimiento de las otredades silenciadas, entre las cuales se puede considerar al sumak kawsay, se plasmó en el caso ecuatoriano, por una resistencia política en los últimos años entre diferentes actores locales que desde las realidades propias de sus territorios se articularon en una disputa con el elemento común que es el reconocimiento de sus formas de vida locales.

\section{EL MOVIMIENTO INDÍGENA, ACTOR CLAVE EN EL SUMAK KAWSAY}

El reconocimiento de ese otro sujeto negado por el proceso de 
colonialidad se observa en el movimiento indígena que busca mantener sus prácticas propias que se reconocen como el sumak kawsay. Esta disputa pasa por diferentes etapas según el contexto histórico-político que se esté estudiando.

El concepto de acción colectiva se refiere a las acciones que los ciudadanos promueven para lograr la defensa de sus intereses en la sociedad, lo que implica una relación entre la organización compuesta por los miembros y la figura del Estado. En el caso ecuatoriano, la relación entre población indígena y Estado ha pasado por diferentes fases, pero el elemento fundamental de la disputa se centra en la idea del Estado Moderno. Esta visión de Estado proviene de un contexto moderno de dominación sobre un territorio donde las cuestiones simbólicas que lo representan no toman en cuenta las características propias de los grupos indígenas del país (Ortiz, 2014). Este autor señala tres tipos de relación del Estado con los pueblos indígenas, a saber: neoindigenismos asociado al desarrollismo (1983-1992), el multiculturalismo asociado al neoliberalismo (1993-2007) y la plurinacionalidad asociada al pos-neoliberalismo (2008-2013). Sin embargo, antes de la primera etapa señalada respecto al desarrollismo ya se habían creado las organizaciones sociales que promovían y defendían la lucha de los pueblos indígenas en las cuales la práctica de resistencia y reconocimiento propio se mantienen. Otro elemento importante en esta disputa pasa por la territorialización que implica una recuperación del territorio a nivel local en cuanto a prácticas económicas y políticas (Carpio, 2016). Por tanto, el territorio es central para poder entender sumak kawsay pues tal es su centralidad que las relaciones y significados que emergen de él en relación con los grupos humanos asentados en dichos territorios van a ser la base para procesos de lucha, resistencia y propuestas políticas que se han ido desarrollando a través de un largo proceso histórico y que en el caso ecuatoriano llega a su auge en la década de los 90.

Por ello, a nivel del Estado la lucha principal se da entre el modelo de Estado Moderno de herencia colonial con un orden social moderno, frente al modelo plurinacional planteado y demandado por los 28 pueblos y 14 nacionalidades del Ecuador, el que fue reconocido en la Constitución de 2008 
(Constitución Ecuador, 2008). En el caso de la resistencia en el territorio de Zhiña, se plasma en la lucha que han llevado a cabo organizaciones de primer grado como la Unión de Comunidades Indígenas del Azuay (UCIA), la que está formada por comunidades y organizaciones indígenas de base, teniendo un margen de acción territorial. La importancia de la presencia de esta organización de primer nivel en Zhiña, es que esta es filial de la ECUARUNARI que representa a los indígenas de la sierra y tiene un margen de acción regional y aglutina a diversas organizaciones de base, lo cual la caracteriza como una organización de segundo grado. A su vez la ECUARUNARI es parte de la CONAIE con un margen de acción nacional. LA CONAIE (Confederación de Nacionalidades Indígenas del Ecuador) se constituyó en 1986 en el marco del primer Congreso de la CONACNIE (Consejo Nacional de Coordinación de Nacionalidades Indígenas) el que fue organizado en 1980 (CONAIE, 2019).

Esta forma de organización del movimiento indígena en el Ecuador, permite que las acciones desde las bases territoriales encuentren un anclaje y apoyo en niveles locales y nacionales, es decir, la identidad local y sus prácticas, son reforzadas desde estructuras que superan a los territorios, en este caso Zhiña. Por lo tanto, a nivel de la organización indígena, la identidad local viene asociada a este sujeto colectivo de tal forma que las prácticas alternativas, si bien son propias de un territorio específico, responden a una propuesta más amplia a nivel país que solo es posible gracias a la coordinación entre los diferentes niveles de organización.

Además de esa disputa con el Estado, se da una tensión en lo que se refiere a la identidad. Para Estermann (1998) y Echeverría (1995) la diferenciación respecto a la construcción de identidad en los contextos europeos y latinoamericanos es bien marcada. Esta diferencia se basa en la dicotomía individuo/grupo y en la oposición del reconocimiento de la identidad, por la no pertenencia a otros grupos, es decir, la identidad como cuestión individual.

Esta construcción de identidad, más propia de contextos latinoamericanos, ayuda a explicar cómo lo indígena y sus organizaciones llegan a convertirse en sujeto político con capacidades propositivas, interpretativas y movilizadores con la categoría de indio inserta en un proceso 
de cambio. Lo indio pasa de ser una categoría utilizada para clasificar a ciertas poblaciones de manera peyorativa a ser un principio de afirmación, creando así una base que ayuda a construir la identidad étnica, la cual permite adhesión y cohesión política y sobre todo, afirma una estructura simbólica del mundo indio que se ha encontrado en constante presión, la que es ejercida por una estructura de poder que no la reconoce (Herrera, 2013).

Esta presión entre el mundo indígena y la estructura es lo que permite que el sujeto político indio (sujeto político étnico) se genere, ya que este planea un proceso de resistencia y constante afirmación de su condición, lo que implica:

Un juego dialéctico entre dos fronteras -la estructura de poder y el mundo indígenaque a lo largo de la historia ha construido, o mejor, ha permitido la formación particular de un sujeto político, el cual ha venido transformándose a lo largo de los cambios políticos y económicos en las relaciones de producción/explotación del desarrollo capitalista (Herrera, 2003, 2).

A lo largo de la historia, desde la llegada de los españoles a lo que se denominó América, esta estructura de poder ha tomado varias formas, pasando desde el sistema de haciendas, la conformación de Estados Nacionales homogeneizantes teniendo como base a la identidad nacional y, por último, el modelo de desarrollo extractivista que instrumentaliza a la naturaleza, lo que ha sido una de las principales concepciones occidentales que tratan de imperar como modelo económico sobre el mundo indígena. Cada una de estas formas ha construido una posición marginada para ser ocupada por el sector indígena, dejando como opción más viable la resistencia ante esta fuerte exclusión y desarme del mundo indígena. Al revisar de forma general cada una de las formas que ha tomado la estructura de poder, se percibe el proceso por el cual el continente americano empieza a ser incluido en una economía mundial que nace en el mismo momento de la Conquista. Ante esta nueva forma o estructura económica es en la que se va insertado el continente y por ende sus pobladores.

Las estructuras económicas vienen acompañadas de una estructura social dentro de la cual es central identificar las fracciones de la clase 
dominante, grupos en ascenso, grupos subordinados y los marginados del sistema económico (Acosta, 2010). A lo largo de la historia del Ecuador en cada una de sus formas económicas siempre uno de los sectores marginados económica y políticamente ha sido el sector indígena, lo que le ha generado una posición crítica hacia este sector ante un sistema que no lo reconoce como sujeto.

Al estar en un estado de negación y exclusión ante las estructuras de poder, el sector indígena entra en un constante estado de desigualdad y pobreza, debido a que las formas que tomarán la estructura de poder toman sentido y llegan a funcionar a costa del desarme del sistema de vida indígena. Esto implica romper con los procesos por los cuales producían y reproducían su vida social. En respuesta a la presión y desarme que las estructuras de poder ejercen sobre el mundo indígena, se genera la construcción del sujeto político étnico, lo cual permite la reafirmación de ellos y de su mundo.

Este proceso de afirmación y construcción de sujeto político que interpela y propone alternativas ante la estructura de poder que lo niega y presiona tiene que ver principalmente con el proceso de resistencia y reafirmación de la identidad que el pueblo indígena ha llevado a cabo desde la llegada del mundo europeo al continente (Herrera, 2003).

Es importante entender entonces que la identidad andina se construye de una forma distinta a la identidad occidental antes mencionada, la que parte del yo para luego formar el nosotros.

...el Yo (noqa) solo existe como contracción del Nosotros (noqanchis), a diferencia del mundo occidental en donde están fundamental y necesariamente separados, primeramente desde lo lingual (en castellano Yo- Nosotros, en inglés I-We, en alemán Ich-Wir, en francés Je-Nous, etc.) y luego por la constitución misma del ser del sujeto en occidente (Herrera, 2003:4).

La construcción del sujeto en el mundo andino solo es posible gracias a la relación con el resto de sujetos que conviven en un mismo espacio (principio de relacionalidad), como lo explica Herrera (2003) su identidad solo posee sentido en tanto esta emerge del Ayllu o la comunidad (del territorio, la tierra). 
El concepto de territorio vuelve a ser central para entender el proceso por el cual lo indígena desarrolla sus procesos de resistencia y de construcción como sujeto político.

Ahora queda explicar cómo el mundo indígena logra resistir ante toda una estructura de poder que lo atraviesa de forma violenta y con el único objetivo de poder insertar a este en el proceso de desarrollo del capital, en sus formas y relaciones de producción. El proceso por el cual el sector indígena logra mantenerse en el tiempo para luego poder construirse como sujeto político es posible según Herrera (2003), debido a que los indígenas en respuesta al nuevo orden que establece la estructura de poder, dividen su vida social en dos espacios de los cuales emergerá su identidad.

\section{PRÁCTICAS Y EXPERIENCIAS, EL ALLI KAWSAY}

Una vez en el campo de estudio es pertinente recalcar que la crítica al modelo clásico de desarrollo se encuentra tanto en los discursos como en las prácticas de formas de vida pese a la existencia de prácticas derivadas del modelo de desarrollo clásico. El concepto de alli kawsay es una expresión:

...kichwa frecuente en la cotidianidad a nivel lingüístico, histórico, espiritual y vivencial, reflejada en actividades agrícolas, políticas, culturales, sociales, socioambientales y alimenticias de las comunidades indígenas; que, transmite y refleja comportamientos éticos y morales como el Ranti Ranti (reciprocidad) heredados de generación en generación (Guandinango \& Carrillo Maldonado, 2013).

También es importante señalar que el concepto de sumak kawsay está relacionado con el de alli kawsay, sin embargo no están implicados directamente. Mientras que el sumak kawsay forma parte de la propuesta de cambio de formas de vida desde una visión más macro hacia lo micro, el alli kawsay "refleja la práctica vivencial cotidiana de los pueblos kichwas" (Guandinango \& Carrillo Maldonado, 2013).

Hablar de territorio implica hablar de autonomía, de apropiación y reapropiación de los lugares de asentamiento de las comunidades. El territorio 
es el elemento central de la vida en el ser humano, toda actividad se desarrolla y toma significado en un espacio particular. Por ello, "para entender la historia de los pueblos indígenas se debe partir de la lucha de las comunas, pueblos y nacionalidades y desde las estructuras de los movimientos sociales" (Inuca Lechón, 2018).

Los valores asignados al territorio no siempre se asientan en la idea de utilidad, pues existen valores que tienen que ver con la historia de la población y con valores de tiempo, es decir, donde se desarrollaron sus experiencias vitales, así como de forma de vida, pues es en ese lugar donde logran el sustento diario y en el cual se desarrollan las relaciones humanas (Gudynas, 2011).

Es desde el territorio donde se construye la práctica identitaria y de resistencia frente a ese modelo que intenta la homogeneización del sistema en un todo integral. Una de las estrategias que se ha utilizado en el mantenimiento de esta noción de un territorio no determinado por la presión de un sistema homogeneizante es la de resistencia, la cual implica "la capacidad de todo sujeto de enfrentar el ejercicio del poder, de intentar salirse del juego, de escabullirse o de hacerle trampa al poder'" (Margarita Del C \& Zárate, 2015: 66). Los levantamientos de los 90 se hacen posibles ya que desde finales del siglo XX surge, dentro de los nuevos movimientos sociales, el movimiento indígena (indígena y campesino-indígena), el cual tiene una lógica que los caracteriza como comunitario-ancestral. Además su anclaje tan fuerte al territorio como base para su identidad y sus formas de organizar la vida colectiva los caracteriza también como movimientos socioterritorial (Mançano Fernades, 2015). Como ya se mencionó, el territorio y la territorialidad es un tema central en la formación de los movientes indígenas, su lucha y demandas de la territorialidad entendida desde los movimientos indígenas será una articulación entre espacio, naturaleza, cultura, política y tiempo social (Flores, 2015).

Los movimientos indígenas toman fuerza como sujetos sociales y políticos que logran poner en debate en el espacio social de países como Ecuador, nuevos temas tales como la creación del Estado Plurinacional 
contrario a la idea del Estado Nación homogéneo occidental. Es importante mencionar que al hablar de un Estado Plurinacional debe estar implícito, como explica Acosta (2009) la idea de nuevas formas de relación entre sociedad y naturaleza, lo que implica nuevas formas de entender el territorio, su manejo por parte de la sociedad y la identidad que nace de esta relación. Además "La idea de plurinacionalidad conlleva a su vez otras ideas: interculturalidad y poscolonialidad." (Santos, 2009), por lo cual esta propuesta del movimiento indígena implica una alternativa al modelo de Estado moderno, permitiéndoles a los pueblos indígenas una mayor autonomía dentro de sus territorios.

Este posicionamiento de los movimientos indígenas como actores locales, nacionales y hasta internacionales, es el resultado de su propio ejercicio de resistencia y construcción como movimientos social y modelo de sociedad (Acosta, 2010).

\section{METODOLOGÍA}

La metodología aplicada en la investigación fue desde el enfoque cualitativo interpretativo, utilizando también técnicas de la Investigación Acción Participativa (IAP), que partiendo del principio de incertidumbre, extrae las lecciones de los sujetos sociales emergentes en la lucha por sus derechos.

Lo importante en este estudio es el reconocimiento de los saberes ancestrales como una herramienta de apropiación y relacionamiento con el entorno, así el diálogo de saberes implica una relación de horizontalidad y respeto entre las diversas culturas que construyen el conocimiento, pues cada una de ellas ha desarrollado estrategias de adaptación entre los seres humanos y la naturaleza, ya que: "Somos lo que hacemos en relaciones para superar lo que creemos que somos... Es decir, somos creatividad relacional, desbordes vitales" (R-Villasante, 2014).

Es necesaria una subversión frente al orden constituido para conocer desde la experiencia lo que significan las luchas sociales por los derechos de los pueblos "dar una vuelta por debajo, para ver los fundamentos, ir más 
allá de la ley" (Ibañez, 1994). Se pretende una observación participante con toda la capacidad creativa sobre aquello que viven, construyen y anhelan las comunidades estudiadas.

Se aplicaron 8 entrevistas a profundidad, cuya muestra fue definida a través de un sociograma elaborado con el cabildo de la comuna, en el que se detectaron las posiciones discursivas por afinidad y práctica de lo que se conceptualizó como sumak kawsay.

Pasos metodológicos en la construcción participativa de la información:

- Transectos: Primeras visitas y reconocimiento de la zona guiados por dirigentes comunitarios, miembros del cabildo y conocedores de la historia de la comuna.

- Observación participante: Asistencia a eventos importantes de la comunidad, tales como: Aniversario de la compra de las tierras comunales, celebración del pawkar raimi (carnaval), asistencia a ritos religiosos tales como misas y bendición de la tierra con la chakana, asistencia a las asambleas del cabildo, con invitación de líderes indígenas nacionales.

- Sociograma: Aplicación de esta técnica para la selección de los actores a entrevistarse, según el poder que representan en la comunidad en cuanto a incidencia en la misma y la cercanía de estos al sumak kawsay (buen vivir en la comuna).

- Entrevistas a profundidad: Se realizaron 8 entrevistas a profundidad a los actores que apoyan el sumak kawsay, así como a quienes no están de acuerdo, por otro lado, se seleccionó según la edad, a jóvenes, y adultos, así como mujeres y hombres para que las opiniones tengan un enfoque de género y según la pertenencia a las diferentes organizaciones comunitarias.

- Elaboración de un diagnóstico: Página Web. Este paso metodológico surgió de la necesidad de la comunidad, y reemplazó a la propuesta 
que tenía el equipo de investigación a través de las 4Ls (Logros, Limitaciones, Lecciones aprendidas y Líneas a Seguir), para lo cual se debían realizar talleres. La elaboración de la página web llevó a una mayor cercanía con los comuneros y el equipo de investigación, muchas veces las reuniones se realizaron en la Universidad de Cuenca, logrando de esa manera confianza en el proceso. La página Web con el breve diagnóstico de la comuna, se presentó en una de las asambleas más importantes de la comuna de Zhiña.

- Devolución de la información: La devolución de la información, fue más allá de la socialización de los resultados de la investigación, ya que la comuna sintió que los productos fueron elementos tangibles útiles a su caminar, tales como: Página Web a través de la cual se comunican con el mundo exterior y se relacionan con los migrantes de la comuna fundamentalmente con aquellos que están en los Estados Unidos de Norteamérica y España; el diagnóstico sirvió para la planificación de las actividades comunitarias.

La confianza y el valor de la palabra entre el equipo de investigación y los miembros del cabildo fueron los mecanismos que permitieron identificar que uno de los elementos alternativos de la comuna, es su estructura organizacional con identidad propia y su permanente lucha social por los derechos, frente a la exclusión y la desigualdad.

En la concepción del tiempo de la cultura kichwa el tiempo es ascendente y en espiral, avanza cerrando círculos y de esta manera va consolidando los procesos. Durante los dos años de investigación el proceso metodológico que ayudó al involucramiento y la participación se puede graficar de la siguiente manera (ver figura 1):

\section{RESULTADOS Y DISCUSIÓN}

Como resultados de la investigación se encuentran dos elementos centrales. Por un lado, está la resistencia de la comuna por conservar sus 
FIGURA 1

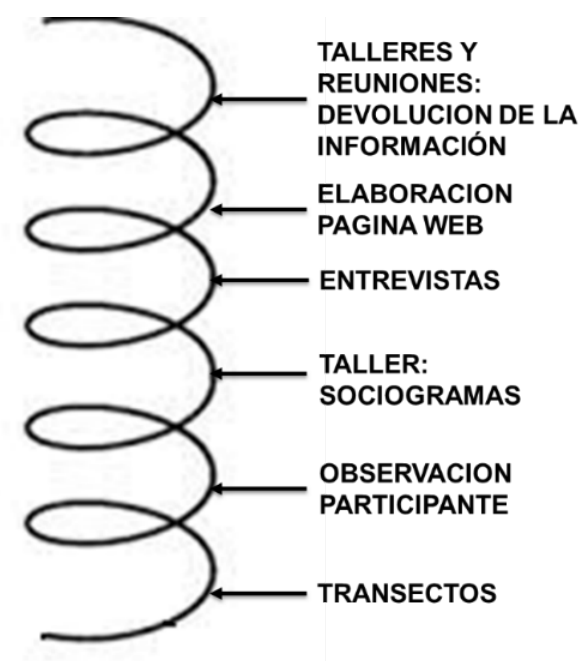

Elaboración: José Astudillo

Banegas.

valores comunitarios, así como la legitimidad de las autoridades locales denominada cabildo lo cual implica el resguardo del suelo como propiedad comunal. Por otro lado, en la comuna se encuentra un grupo de comuneros que se denominan "los colonos", compuesto fundamentalmente por emigrantes retornados que no comparten los valores históricos de la comuna ni reconocen la autoridad política del cabildo. El discurso de los colonos es defensor del modelo de desarrollo clásico, lo cual supone una confrontación de valores entre miembros de la comuna que se orienta hacia prácticas cotidianas opuestas entre comuneros y colonos.

La importancia de la lucha en defensa de los derechos es el hilo conductor de la historia de Zhiña. No es una lucha por conseguir los servicios básicos que ofrece el mundo del desarrollo, sino por el acceso a los derechos 
básicos fundamentales en la defensa y promoción del agua, alimentación, educación, y territorio, "la garantía de los derechos con identidad propia" (Vega, 2016:127). Identidad propia conformada desde el territorio local, pues está asociada a la existencia de derechos colectivos y por tanto sujetos colectivos que mantienen una vinculación especial con el territorio. Por ello el desarrollo de proyectos y acciones sociales que ayudan a la revalorización de los saberes ancestrales como la medicina, ha sido una característica central e importante en la comuna de Zhiña.

La compra de la hacienda en la década de los años treinta, es uno de los hitos más importantes de liberación, cuyo resultado fue desatarse del yugo que representaba el sistema hacendatario como eje de acumulación sustentado en la explotación a los indígenas (Larrea, 2006; Acosta, 2012). Una antigua dirigente de Zhiña narra esta historia socioeconómica de la comuna:

Cuando empezaron a comprar la tierra, también lucharon nuestros papás, ya me acordaba como ellos han luchado, como han andado para comprar la tierra, para tener la tierra propia, para ya no ser esclavos de nadie. Antes mi suegra conversaba, diciendo que ellas han sido huasipungeras, que los papás han sido huasipungeros, peones (Morocho J., 2016).

En la compra de la hacienda y la gestión comunitaria del cabildo para mantener la cultura, el idioma y lograr servicios como agua potable, luz eléctrica, alcantarillado, carreteras de integración entre comunidades, colegio intercultural bilingüe, etc, está la expresión alli (bueno) al que se refieren frecuentemente los actores de la comuna Zhiña, "incluimos el adjetivo alli porque las comunas indígenas de Nabón usan frecuentemente la expresión alli kawsay" (Vega, 2016: 128). Fue a partir de la compra de la Hacienda Zhiña en el año 1939 cuando se crea el primer cabildo y se escritura la tierra de manera comunal. Las autoridades del cabildo son representadas solamente por los miembros que comparten los valores de la historia de la comuna y muchos de sus miembros han sido y son representantes de organizaciones como la UCIA y la ECUARUNARI.

Más tarde, en la década de los 60-70, la lucha continúa en el marco de 
la reforma agraria: “...empezamos a luchar para defender la reforma agraria, defender el agua, defender el terreno, eso sí me duele, ¡eso si te digo yo!, ¡vivo reclamando!"” (Morocho J. , 2016). En los años 90 Zhiña se posiciona como uno de las comunas más importantes en la lucha por los derechos colectivos, a través de la UCIA, como afirma una luchadora: "Todo gobierno hemos respetado, hemos dicho esto se hace y tiene que hacerse, hemos salido a las lomas, hemos peleado con los policías, hemos enfrentado y ganábamos todos nuestros derechos, [...] porque los indígenas también tenemos derechos" (Morocho J. , 2016). Los derechos colectivos indígenas han sido representados por el Estado Plurinacional, por ello que la idea de lo plurinacional esté unida a los derechos colectivos indígenas. El reconocimiento de los derechos colectivos indígenas se representa en la figura del Estado Plurinacional, por ello que esta idea vaya de la mano de lo plurinacional. Recuperar la territorialidad implica el reconocimiento del derecho al territorio, a su propia educación, a su propio idioma, etc.

Los indígenas de Zhiña han defendido el páramo, la tierra, el agua, y han luchado en favor de la vialidad, defendiendo a las instituciones de carácter local en contra del centralismo gubernamental, motivo por el cual existen dirigentes encarcelados: Así comenta la alcaldesa del cantón Nabón: “Uno ya está sentenciado a 2 años. Un mayorcito de 80 años, ¿qué puede hacer un mayorcito de 80 años? Ustedes podrán imaginar. [...] por estar reclamando en la vía pública, por algunos temas que no estamos de acuerdo" (Quezada, 2016).

El acompañamiento de las Misioneras Lauritas y la influencia de la Teología de la liberación, entre otras, fueron muy importante para la comuna. En este proceso de acompañamiento pastoral, tanto la recuperación de la identidad, así como las culturas ancestrales y la lucha por la igualdad, son una constante (Vega, 2016).

En la comuna y en el cantón de Nabón se reconoce este acompañamiento pastoral de liberación: "Por eso acá, nosotros hablamos [...] la iglesia y el pueblo, dos partes, pero caminamos ambos, en un mismo cuerpo se camina con ambos pies, pero el mismo camino hacemos nosotros" (Sagbay, 2016). 
La iglesia se ha integrado a la comunidad desde la pastoral indígena, se observa incluso muchas prácticas religiosas, donde la ritualidad cristiana e indígena conviven y se respetan mutuamente: "Si usted va a una misa, primero es el ritual y luego es la misa inclusive en las primeras comuniones, en las confirmaciones, primero es la limpia, el ritual y luego va la confesión"(Quezada, 2016). La ritualidad cristiana que proviene de la vertiente de la teología de la liberación y de la teología indígena, es muy respetuosa de la cultura como espacio de identidad y la revalorización de los saberes ancestrales, así como la espiritualidad propia del pueblo indígena como fuente de liberación.

Los sentidos de liberación como pueblo, identidad de personas y seres humanos iguales, la redistribución y el compartir de los bienes y el cuidado de la naturaleza como algo sagrado, son los puntos de convergencia entre la ritualidad indígena y la cristiana en la comuna de Zhiña.

La lucha por los derechos colectivos, se promovió también desde el apoyo de algunas instituciones que implementaron proyectos con la participación de la comunidad. En el cantón de Nabón desde 1997 a través del proyecto de desarrollo participativo, a cargo de la Oficina de Investigaciones Sociales y del Desarrollo (OFIS) y la Central Ecuatoriana de Servicios Agrícolas (CESA) y más tarde en el 2003 con fondos de PROLOCAL, se desarrollaron una serie de emprendimientos que cambiaron la vida de los habitantes de la zona, fortaleciendo la organización a nivel político y técnico, y la introducción de nuevos cultivos y alternativas productivas (Vega, 2016).

En la implementación de proyectos y programas de desarrollo, son las mujeres quienes se han involucrado, logrando así un empoderamiento social:

Está la organización de productores de leche, están las cajas de ahorro y crédito, si no estoy mal, es el mayor número de cajas que tenemos en la zona indígena, las cajas son llevadas por mujeres, las cajas de ahorro y crédito manejan capitales (Quezada, 2016).

A través de los proyectos, la comuna ha mejorado su alimentación, el autoconsumo y soberanía alimentaria, aunque en la actualidad uno de los problemas que enfrenta la producción es la falta de acceso a mercados limpios. 
Se reconocen también las políticas de gobierno en la construcción de vías y el asfalto desde Zhiña Centro hacia Cuenca, que ha mejorado las condiciones de comercialización de sus productos.

Los cultivos tradicionales y los nuevos cultivos, han dinamizado la comunidad, más allá del maíz, trigo, papas, ajo, mellocos, mashua, zambos, zapallos, ahora con los invernaderos de frutillas, la horticultura orgánica y la ganadería: "la implementación de todo este proceso de capacitación; impulso de nueva tecnología, de mejoramiento tecnificado de riego, fueron mejorando sus fincas, ahora en sus fincas ya vemos diferente producción: manzanilla, plantas aromáticas[...] lácteos en Zhiña" (Quezada, 2016).

Un acierto del Proyecto Nabón fue la alianza con la Cooperativa Jardín Azuayo para promover los microcréditos y la inversión en Zhiña; la cooperativa se ha convertido en la instancia de confianza de los habitantes, para recibir las remesas de los migrantes. Tan importante es esta alianza que el cabildo le considera como un actor participante en sus reuniones y consejero para la toma de decisiones. El representante de la cooperativa afirma que su relación en muy estrecha para la implementación de emprendimientos:

Aquí, hemos estado ayudando, [...] con lo que es la producción de tomates. [...] Hemos ayudado en lo que es producción de frutillas, también han estado sacando un poco, pero últimamente, [...] estamos colocando bastantes créditos para lo que son la ganadería [...] se ve que ahora hay buena producción de leche (Morocho J., 2016).

La lucha por los derechos sociales, económicos y culturales, ha pasado del discurso a la acción y en este proceso algunas $\mathrm{ONG}^{\prime}$ s e iglesia, comprometidas con el cambio social, han logrado desde el desarrollo local, impulsar la comunidad como una instancia política que va consolidando un proyecto alternativo, al paternalismo (Guerreo \& Ospina, 2003; Tortosa, 2011).

Hoy la comuna de Zhiña, es una de las organizaciones indígenas más fuertes de la zona, que organizadamente defiende los derechos de los pueblos, frente a la dominación del Estado y los gobiernos de turno que no han logrado romper con la estructura de desigualdad y exclusión, sin embargo, existe 
también una disputa interna entre la población comunal y los colonos.

Este conflicto entre colonos e indígenas es importante en la comuna y en él se visualiza la convergencia de varias identidades y religiones, pues si bien la identificación religiosa mayoritariamente es católica, se encuentra la presencia de otras iglesias del mismo cristianismo que se instalaron en la comuna de Zhiña. Estas iglesias llegaron cuando se empezó a dar el retorno de los migrantes autodenominados colonos. Una nueva identidad religiosa en la comuna de Zhiña es la de los cristianos anglicanos. Debido al conflicto con los emigrantes retornados que no reconocen la autoridad del cabildo quien mantiene una fuerte relación con la Iglesia Católica, los colonos fundaron la asociación "Los colonos de la Hacienda de Zhiña" y establecieron una alianza con la Iglesia Anglicana. Las dos identidades religiosas son, por un lado, los emigrantes retornados que se autodenominan colonos y ya no se sienten indígenas, y los católicos que se han convertido en anglicanos.

Van a tener algunas impresiones frente a Zhiña con el tema de la aculturización con el tema de los colonos que fueron algún rato migrantes que regresaron a la zona y que ha habido una separación de la comuna con esta organización de colonos migrantes (Quezada, 2016).

Los colonos que se originaron a partir del regreso de los emigrantes de la comuna, se encuentran cada vez más en la población descendiente de esos ex migrantes que nunca han salido de la comuna. La aculturación de esta población se produce en tanto se deja de utilizar la vestimenta, de participar en actividades de la comuna como las mingas y de usar el idioma.

La tierra es el fundamento de la identidad indígena en la comuna de Zhiña. Defender el territorio comunal, las formas de solidaridad y reciprocidad en el trabajo hace la diferencia de otros indígenas denominados los colonos, para quienes el desarrollo económico y la propiedad individual, es lo central para modernizarse y alcanzar el progreso.

\section{CONCLUSIONES}

Cuando se trata de encontrar prácticas alternativas al desarrollo clásico, 
se debe plantear un análisis acerca de cómo convergen estilos de vida locales propios de grupos sociales indígenas con elementos que originalmente le son ajenos.

Plantear que desde los territorios se proponen alternativas reales implica una visión reduccionista sobre una realidad social que resulta más compleja. Es por ello que más allá de las prácticas que dan cuenta de una disputa constante entre formas de vida local y aquellas que llegan desde otros lugares, es necesario comprender cómo se produce la convergencia entre ellas. A nivel político se encuentran dos formas de gobiernos locales; cabildo con territorio comunal versus parroquias (división administrativa a nivel país). Una de las demandas de los colonos es la conversión de la comuna de Zhiña en parroquia con el fin de poder dividir el territorio. La compra de la hacienda en el caso de Zhiña, supuso una negociación con el Estado, que se ha mantiene en la consecución de servicios básicos como agua potable, alcantarillado, escuela intercultural bilingüe, etc. a partir de la interacción con gobiernos políticos de tipo municipal y regional. La incorporación de la Iglesia como actor de apoyo en sus demandas propias para la implementación de proyectos y programas de desarrollo como las cooperativas, suponen la coexistencia de prácticas religiosas ancestrales en el seno de la Iglesia y la aceptación del funcionamiento de mecanismos administrativos regionales y estatales.

Las formas de vida se refieren a las relaciones entre personas, autoridades y sistema administrativo, y representan una convergencia entre imaginarios sociales ancestrales y realidad operativa entendiendo que la consecución de derechos y reconocimientos propios se da a partir de la aceptación del funcionamiento del sistema administrativo y político central.

La negociación a nivel local desde el brazo político que articula a la comuna de Zhiña, ha sido el hito principal en el último tiempo y continúa en la actualidad, pues en conflictos de identidad como la división entre indígenas y colonos, son las organizaciones políticas como la UCIA o el cabildo de la comuna de Zhiña, quienes en la práctica no aceptan puntos de vista diferentes a su principios políticos por parte de la población colona y es por este motivo, que ellos no forman parte de este tipo de organizaciones. Que los colonos no 
formen parte de este tipo de organizaciones supone una exclusión también del alli kawsay, ya que estas organizaciones están presentes en las prácticas del territorio tales como mingas, celebraciones religiosas y festividades de las cuales no forman parte los colonos.Un análisis teórico que profundice acerca de qué manera se da esta convergencia a partir de elementos internos y externos al territorio estudiado no solo en las relaciones entre población, autoridades, sistema administrativo y político, sino también a nivel económico, prácticas culturales, ambientales, etc, sería un aporte interesante que ayude a comprender en un mayor rango analítico, cómo se producen alternativas al modelo de desarrollo desde la cultura local indígena.

\section{REFERENCIAS}

Acosta, Alberto (2010) El Buen Vivir en el camino del post-desarrollo. Una lectura desde la Constitución de Montecristi. Policy Paper 9.

Acosta, Alberto (Marzo de 2016). Entrevista a profunidad a expertos temáticos realizada por A.M.A. Ferrer, P. Paño, \& J. Astudillo.

Carpio, Patricio. (9 de Junio de 2016). Entrevista a profundidad a expertos temáticos realizada por A.M.A Ferrer, P. Paño, \& J. Astudillo.

CONAIE. (9 de marzo de 2019) Rcuperado de: https://conaie.org. Obtenido de https://conaie.org/2014/07/19que-es-la-conaie-2/

Constitución de la República del Ecuador (2008). Artículo 71, 72, 73, 74. Capítulo Tercero.

Cubillo-Guevara, A. P., \& Hidalgo-Capitán (2015).El sumak kawsay genuino como fenómeno social amazónico ecuatoriano. OBETS. Revista de Ciencias Sociales, 10 (2) 301-303.

Dussel, E. (2008). El encubrimiento del Otro. Hacia el origen del "mito de la Modernidad”. La Paz: Biblioteca Indígena.

Echeverría, B. (1995). Las ilusiones de la modernidad. Ensayos. México: UNAM/ El equilibrista.

Estermann, J. (1998). Filosofia Andina. Estudio Intercultural de la sabiduría autóctona andina. Quito: ABYA YALA. 
Ferreira Vázquez, Miguel Ángel (2005). La reflexividad social transductiva: la constitución práctico-cognitiva de lo social y de la sociología. Revista Crítica de Ciencias Sociales y Jurídicas 11 (1). 287-303 .

Guandinango, Y., \& Carrillo Maldonado, P. (Enero de 2013). Sumak Kawsay y Alli Kawsay. El proceso de Institucionalización y la Visión Andina. SSRN. Obtenido de https://ssrn.com/abstract $=1980120$

Gudynas, E. (2010). Desarrollo sostenible: una guía básica de conceptos y tendencias hacia otra economía. Otra Economía. Revista Latinoamericana de economía social y solidaria, 4 (6)

Gudynas, E. (2011). Buen Vivir: Germinando alternativas al desarrollo. América Latina en Movimiento., ALAI Obtenido de https://www. alainet.org/es/active/48052

Herrera, S. (2003). El proceso de construcción de la identidad política del movimiento indígena ecuatoriano. Buenos Aires. CLACSO.

Ibañez, J. (1994). Por una sociología de la vida cotidiana. México: Siglo XXI.

Inuca Lechón, J. B. (2018). Llaktapura sumak kawsay/vida plena entre pueblos. Un concepto emancipatorios del Ecuador. Mecila: Working Paper Series.

Joas, H., \& Knöbl, W. (2016). Teoría Social. Veinte lecciones introductorias. Madrid: Akal.

Max-Neef M., E. A. (1993). El desarrollo a Escala Humana. Barcelona. ICARIA.

Morocho, J. (24 de Julio de 2016). Entrevista a profundidad de actores convivenciales comuna de Zhiña realizada por J. Martines, \& M. Cobos.

Morocho, J. (1 de Junio de 2016). Entrevista a profundidad de expertos convivenciales comuna de Zhiña realizada por A.M.A. Ferrer \& J. Martines

Ortiz Tirado, P. (2014). Políticas estatales, territorios y derechos de los pueblos indigenas en Ecuador (1983-2012). Buenos Aires: CLACSO, Consejo Latinoamericano de Ciencias Sociales.

Quezada, M. (1 de Julio de 2016). Entrevista a profundidad de expertos 
territoriales comuna Zhiña realizada por J. Astudillo.

Restrepo, E., \& Rojas, A. (2010). Inflexión decolonial: fuentes, conceptos y cuestionamientos. Popayán: Universidad del Cauca.

R-Villasante. (2014). Redes de vida desbordantes: Fundamentos para el cambio desde la vida cotidiana. Madrid: Catarata.

Sagbay, A. (1 de Junio de 2016). Entrevista a profundidad de expertos convivenciales comuna de Zhiña realizada por J. Astudillo. (J. Astudillo, Entrevistador)

Vega, F. (2014). El Buen Vivir-Sumak Kawsay en la Constitución y en el PNVB 2013-2017 del Ecuador. OBETS. Revista de Ciencias Sociales, $9(1), 167-194$.

Vega, F. (2016). El Buen Vivir en el territorio y comunidades del cantón Nabón 2011 - 2015. Cuenca: Pydlos Ediciones. 